\title{
Erucin Inhibits Osteoclast Formation via Suppressing Cell-cell Fusion Molecule DC-STAMP Without Influencing Mineralization by Osteoblasts
}

\section{Tomohiro Takagi}

Tohto University

Hirofumi Inoue

Tokyo University of Agriculture

Shungo Fujii

Hokkaido Bunkyo University

Nobuyuki Takahashi

Tokyo University of Agriculture

Mariko Uehara ( $\nabla$ mari@nodai.ac.jp )

Tokyo University of Agriculture

Research note

Keywords: Erucin, isothiocyanate, osteoclast differentiation, osteoclast cell fusion, DC-STAMP

Posted Date: August 24th, 2021

DOl: https://doi.org/10.21203/rs.3.rs-840393/v1

License: (c) (i) This work is licensed under a Creative Commons Attribution 4.0 International License.

Read Full License

Version of Record: A version of this preprint was published at BMC Research Notes on March 16th, 2022.

See the published version at https://doi.org/10.1186/s13104-022-05988-3. 


\section{Abstract}

Objective: Erucin (ERN), an isothiocyanate, is derived from the vegetable arugula. Although ERN has antitumor and antioxidant activity, the effect of ERN on osteoclast and osteoblast differentiation is not well documented. In this study, we evaluated the effects of ERN on osteoclast and osteoblast differentiation in vitro.

Results: ERN significantly reduced the formation of $1 \mathrm{a}, 25(\mathrm{OH})_{2} \mathrm{D}_{3}$-induced tartrate-resistant acid phosphatase (TRAP)-positive cells at non-cytotoxic concentrations. Furthermore, ERN downregulated the mRNA expression of osteoclast-associated genes, such as nuclear factor of activated $T$ cells cytoplasmic-1, TRAP, and cathepsin K. In addition, ERN suppressed dendritic cell specific transmembrane protein (DC-STAMP), which encodes cell-cell fusion. However, ERN did not affect mineralization by osteoblasts. Thus, our data suggest that ERN may attenuate osteoclastic bone resorption by inhibiting multinucleation of mononuclear pre-osteoclasts and by suppressing mRNA expression of DC-STAMP in bone marrow cells without influencing mineralization by osteoblasts.

\section{Introduction}

Bone continuously repeats bone resorption and bone formation by osteoblasts [1]. Bone resorption and formation are stable under physiological conditions. However, when this balance is disturbed, bone structure and function become abnormal, resulting in various skeletal diseases, such as osteoporosis, rheumatoid arthritis, and periodontitis [2, 3]. In many cases, the activation of osteoclasts results in bone fragility. Therefore, it is important to identify the molecules that regulate osteoclast or osteoblast differentiation in maintaining bone homeostasis.

Osteoclasts are multinucleated cells that play key roles in mineralized bone matrix degradation. They are formed by fusing mononuclear precursors of the monocyte/macrophage lineage. On the other hand, osteoblasts are mononuclear cells, differentiate from mesenchymal cells, and are involved in the regulation of bone metabolism by synthesizing bone matrix, which becomes progressively mineralized. Osteoblasts are responsible for depositing hydroxyapatite and calcium phosphate crystals. Receptor activator of nuclear factor K-B ligand (RANKL) produced by osteoblasts acts as an essential modulator of osteoclast differentiation and activation by directly binding to its receptor, RANK, which is expressed on osteoclast precursors and mature osteoclasts. RANKL specifically binds to its receptor, RANK, and regulates transcription factors such as c-Fos (a member of the dimeric transcription factor AP-1) and nuclear factor of activated T cells cytoplasmic 1 (NFATc1) [4]. In particular, NFATc1 are crucial activators of osteoclast-associated genes and activates target genes such as tartrate-resistant acid phosphatase (TRAP) and cathepsin K (Ctsk). [5]. Then, osteoclast cells undergo fusion via cell-cell fusion molecules, such as dendritic cell-specific transmembrane protein (DC-STAMP) and osteoclast stimulatory transmembrane protein (OC-STAMP). These molecules are the main factors involved in the regulation of bone resorption, and DC-STAMP or OC-STAMP-deficient cellsare not be able to develop into 
multinucleated osteoclasts [6, 7]. Importantly, osteoclast cell fusion by DC-STAMP and OC-STAMP activation is essential for the multinucleation of pre-osteoclasts.

In cruciferous vegetables, many different glucosinolates yield isothiocyanate (ITC). Erucin (ERN) (Fig. 1A) is derived from arugula, a cruciferous vegetable, and induces apoptosis in several cancer cell lines $[8,9]$. The anticancer activity of ITC is known to be mediated, at least in part, by induction of apoptosis and is associated with the presence of $\mathrm{a}-\mathrm{N}=\mathrm{C}=\mathrm{S}$ moiety. Recently, we demonstrated that sulforaphane (SFN) and sulforaphene (SFE) inhibit osteoclast differentiation by suppressing the cell-cell fusion molecules DC-STAMP and OC-STAMP $[10,11]$. SFN is the most extensively studied ITC in cruciferous vegetables, and ERN, being closely related to SFN, has also received attention because of its similar structure to SFN. However, since ERN does not contain oxidized sulfur, it might be expected that its biological effects would be weaker than those of SFN and SFE. Furthermore, the effects of ERN on bone metabolism have not yet been documented. In the present study, we investigated the effects of ERN on pre-osteoclast multinucleation and osteoblast differentiation in bone marrow cells (BMCs), which are similar to in vivo conditions.

\section{Materials And Methods}

\section{Materials}

ERN was purchased from Cayman Chemical (Ann Arbor, MI, USA). $1 \mathrm{a}, 25(\mathrm{OH})_{2} \mathrm{D}_{3}$ was obtained from Sigma-Aldrich (St. Louis, MO, USA). Osteoblast-inducer reagent was purchased from Takara Bio Inc. (Shiga, Japan). Soluble RANKL (sRANKL) was purchased from R\&D Systems (Minneapolis, MN, USA). aminimal essential medium (a-MEM) (phenol red-free) was obtained from Gibco BRL/Invitrogen (Carlsbad, CA, USA).Fetal bovine serum (FBS) was obtained from Biowest (Nuaillé, France). Cell Counting Kit-8 (CCK8) was purchased from Dojindo (Kumamoto, Japan).

\section{Cell culture}

BMCs were obtained from the femur and tibia of 8week-old male ddY mice. Male ddY mice were purchased from Japan SLC Co. (Hamamatsu, Japan). The mice were fed AIN-93G diet and given distilled water freely for three days as the acclimatization period. The mice were euthanized with an intraperitoneal injection of anesthesia (medetomidine hydrochloride $0.3 \mathrm{mg} / \mathrm{kg}+$ midazolam $4 \mathrm{mg} / \mathrm{kg}$ + butorphanol tartrate $5 \mathrm{mg} / \mathrm{kg}$ ) followed by cervical dislocation. BMCs were isolated from femora and tibias, and were collected by centrifugation at 6,000rpm for $20 \mathrm{sec}$ in $2.0 \mathrm{~mL}$ microcentrifuge tubes, followed by a-MEM. In the experiment, BMCs from two mice were mixed and used as the BMC samples. BMC samples were randomly divided into two groups, a control group and an ERN treated group. The animal protocols and procedures used in this study were approved by the Tokyo University of Agriculture Animal Use Committee, and mice were maintained in accordance with the guidelines of the University for the care and use of laboratory animals. Marrow cells were flushed from bones, and cells were cultured in a-MEM (phenol red-free) supplemented with $10 \%$ FBS, $100 \mathrm{U} / \mathrm{mL}$ penicillin, and $100 \mu \mathrm{g} / \mathrm{mL}$ streptomycin (Gibco BRL/Invitrogen) at $37^{\circ} \mathrm{C}$ in a humidified $5 \% \mathrm{CO}_{2}$ atmosphere. RAW264.7 cells, mouse 
macrophage/monocytes, were obtained from the American Type Culture Collection (Manassas, VA, USA) and were cultured in a-MEM supplemented with $10 \% \mathrm{FBS}, 100 \mathrm{U} / \mathrm{mL}$ penicillin, and $100 \mu \mathrm{g} / \mathrm{mL}$ streptomycin (Gibco BRL/Invitrogen) at $37^{\circ} \mathrm{C}$ in a humidified $5 \% \mathrm{CO}_{2}$ atmosphere.

\section{Cytotoxicity assays}

To evaluate the effect of ERN on the cell viability of BMCs, cytotoxicity assays were performed using the CCK-8. Briefly, BMCs $\left(1 \times 10^{5}\right.$ cells/well) were cultured in 96-well plates. Then, treated with the presence or absence of ERN $(0.01-5 \mu \mathrm{M})$ for 6 days in a-MEM containing $10 \%$ FBS. The effect of ERN on cell viability was calculated as percent cell viability, with ERN-untreated cells set at $100 \%$.

\section{Osteoclast differentiation assay}

To form multinucleated osteoclasts, BMCs were differentiated into osteoclasts using $1 \mathrm{a},-25(\mathrm{OH})_{2} \mathrm{D}_{3}$. BMCs $\left(1 \times 10^{6}\right.$ cells/well) were treated with $10^{-8} \mathrm{M}$ of $1 \mathrm{a},-25(\mathrm{OH})_{2} \mathrm{D}_{3}$ to induce differentiation in the presence of ERN at a concentration of $0-1 \mu \mathrm{M}$ in a 96-well plate for 6 days. After 6 days of incubation, the cells were fixed in $10 \%$ formaldehyde and then stained for TRAP, a marker enzyme of differentiated osteoclasts. TRAP-positive cells with $\geq 3$ nuclei were scored as differentiated osteoclasts. The effect of ERN on osteoclast differentiation was calculated as the osteoclast formation rate, with ERN-untreated control cells set at $100 \%$.

\section{Real-time PCR analysis}

BMCs $\left(1 \times 10^{7}\right.$ cells/well) were seeded in a 24-well plate, treated with $10^{-8} \mathrm{M}$ of $1 \mathrm{a},-25(\mathrm{OH})_{2} \mathrm{D}_{3}$ and various concentrations of ERN $(0.1$ and $1 \mu \mathrm{M})$ for 6 days. Total RNA was isolated from BMCs using Sepasol-RNA I Super G (Nacalai Tesque, Tokyo, Japan). Then, Single-stranded cDNA was synthesized from total RNA using reverse transcriptase (Takara Bio Inc.). Real-time PCR was performed using the THUNDERBIRD qPCR Mix (Toyobo, Osaka, Japan) and results were analyzed using the ABI StepOnePlus System (Applied Biosystems, Foster City, CA, USA). Real-time PCR was performed using the following primers: c-Fos, 5'GAGTGATGCCGAAGGGATAA-3' (forward) and 5'-GAGAAGCATTCCGGTCAGAG-3' (reverse); NFATC1, 5'GCTTCACCCATTTGCTCCAG-3' (forward) and 5'-ATGGTGTGGAAATACGGTTGGTC-3' (reverse); TRAP, 5'ACTTCCCCAGCCCTTACTAC-3' (forward) and 5'-TCAGCACATAGCCCACACCG-3' (reverse); Ctsk, 5'CCAGTGGGAGCTATGGAAGA-3' (forward) and 5'-CTCCAGGTTATGGGCAGAGA-3' (reverse); DC-STAMP, 5'TCCTCCATGAACAAACAGTTCCA-3' (forward) and 5'-AGACGTGGTTTAGGAATGCAGCTC-3' (reverse); OCSTAMP, 5'-TGTCCTACAGTGCAGCCAAC-3' (forward) and 5'-TCTCCTGAGTGATCGTGTGC-3' (reverse); $\beta$ Actin, 5'-TGTCCACCTTCCAGCAGATGT-3' (forward) and 5'-AGCTCAGTAACAGTCCGCCTAGA-3' (reverse). All reactions were normalized to the housekeeping gene $\beta$-actin $(A C T B)$.

\section{Mineralization analysis}

BMCs $\left(1 \times 10^{6}\right.$ cells/well $)$ were seeded in a 96 -well plate for $24 \mathrm{~h}$. Cells were then cultured with various concentrations of ERN $(0-1 \mu \mathrm{M})$ in the presence of osteoblast-inducer reagents (ascorbic acid, $\beta$ - 
glycerophosphate, and hydrocortisone) for 15 days. After incubation, the cells were fixed and stained with $1 \%$ alizarin red. For quantitative analysis, cells were destained with ethylpyridinium chloride and transferred to a 96-well plate to measure optical absorbance at $570 \mathrm{~nm}$ using a microplate reader. The effect of ERN on osteoblast differentiation is expressed as the degree of mineralization, with ERNuntreated cells set at $100 \%$.

\section{Statistical analysis}

Results were presented as means \pm SE of measurements performed on 3-6 cultures in each experimental or control group (there was no exclusion for any experimental unit.). All experiments were independently analyzed at least three times to confirm the results. For statistical significance, multiple comparisons were performed using Tukey's test, after one-way analysis of variance (ANOVA). Statistical significance was set at $P<0.05$.

\section{Results}

\section{Effects of ERN on osteoclast differentiation}

We evaluated the cytotoxic effects of ERN in BMCs close to in vivo conditions using the CCK-8 assay. Low-dose ERN exerted no cytotoxicity, but decreased cell viability at a concentration of $5 \mu \mathrm{M}$ (Fig. 1B). These results indicated that the maximum concentration of ERN used in our subsequent experiments (1 $\mu \mathrm{M})$ had no cytotoxic effects toward BMCs. Next, to examine the effects of ERN on pre-osteoclast multinucleation, cells were incubated with ERN in the presence of $1 a, 25(\mathrm{OH})_{2} \mathrm{D}_{3}$ (Sigma-Aldrich). Compared to that reported for $1 \mathrm{a}, 25(\mathrm{OH})_{2} \mathrm{D}_{3}$-treated cells, ERN decreased the rate of multinucleated osteoclast formation (Fig. 1C). It has been suggested that ERN inhibits pre-osteoclast multinucleation below cytotoxic concentrations.

\section{Effects of ERN on the expression of osteoclast-associated genes}

We examined the effects of ERN on mRNA expression levels of osteoclast-associated genes such as $c$ Fos, NFATC1, TRAP, Ctsk, DC-STAMP, and OC-STAMP, using real-time PCR. Compared to that in $1 \mathrm{a}, 25(\mathrm{OH})_{2} \mathrm{D}_{3}$-treated cells, ERN suppressed mRNA expression levels of NFATc1, TRAP, Ctsk, and DCSTAMP but not $c$-Fos and OC-STAMP (Fig. 2A-F).

\section{Effects of ERN on osteoblast differentiation}

To examine the effects of ERN in mineralization of osteoblast, BMCs were exposed to ERN during osteoblast formation. However, there was no difference in Alizarin red staining activities compared to ERN untreated cells (Fig. 3A). In addition, similar results were obtained from the quantitative analysis of alizarin staining activity (Fig. 3B).

\section{Discussion}


In this study, ERN significantly inhibited osteoclast-differentiation and the expression of the osteoclast cell-fusion associated genes. Initially, we evaluated the effect of ERN on multinucleation of preosteoclasts using BMCs. As a result, ERN inhibited the multinucleation of pre-osteoclasts at non-cytotoxic concentrations. Furthermore, our results indicated that ERN suppresses the expression of the osteoclast differentiation-associated genes NFATC1, TRAP, Ctsk, and DC-STAMP but did not affect C-Fos and OCSTAMP expression. Multinucleated osteoclasts are derived from the fusion of monomeric osteoclasts, and fusion is considered an indispensable process for pre-osteoclast multinucleation and absorption of monomeric osteoclasts. Additionally, according to a previous study, targeted inhibition of DC-STAMP by siRNAs and specific antibody markedly suppressed the multinucleation of pre-osteoclasts [12]. Our data suggest that the inhibitory effect of ERN on the multinucleation of pre-osteoclasts can be attributed to the suppression of the cell-cell fusion molecule DC-STAMP. Additionally, the effects of ERN on cell viability and pre-osteoclast multinucleation of RAW 264.7, osteoclast precursor cells were similar to those observed in BMCs (Fig. S1). On the other hand, we examined the effects of ERN on bone formation in primary osteoblast cultures. Mineralization nodules are biomarkers that determine osteoblast maturation and can be detected by Alizarin staining. When BMCs were cultured with osteoblast-inducer reagent, alizarin-stained mineralized bone nodules were detected on day 15. ERN did not inhibit the formation of mineralized bone nodules. These data suggest that ERN has very little effect on osteoblast mineralization, although ERN inhibits pre-osteoclast nucleation. Collectively, specific inhibition of osteoclast differentiation only may improve bone metabolism without affecting normal bone formation by osteoblasts. According to previous reports, many beneficial effects of ERN are due to its antioxidant and anti-cancer properties [13]. Osteoclasts produce reactive oxygen species (ROS). Free radicals are known to play important roles in osteoclast differentiation through activation of RANKL/RANK signaling [14]. Therefore, the antioxidant activity of ERN may be effective in inhibiting pre-osteoclast multinucleation. However, the inhibitory effect of ERN on pre-osteoclast multinucleation was weaker than that of SFN and SFE, as previously reported $[10,11]$. Harris et al. suggested that both SFN and ERN increase the expression of multidrug resistance protein 1 to a similar extent, although the effect of SFN was substantially greater than that of ERN [15]. Furthermore, other studies have shown that ITCs with oxidized sulfur are the most efficient inducers of apoptosis and the biological activities of ITCs might be affected by the oxidation state of sulfur involving the side chains of such materials [16].

\section{Conclusion}

In the present study, we demonstrated that ERN plays a novel role in inhibiting multinucleation of preosteoclasts by downregulating DC-STAMP. Notably, this is the first evidence that ERN inhibits multinucleation of pre-osteoclasts by suppressing cell-cell fusion without influencing mineralization in osteoblasts.

\section{Limitations}


The precise molecular mechanisms by which ERN improves bone metabolism in vivo are still unclear. Further studies are required to clarify the mechanisms by which ERN improves bone resorption in vitro and osteoporosis in vivo.

\section{Abbreviations}

Erucin; ERN: sulforaphane; SFN: sulforaphane; SFE: Isothiocyanate; ITC: Receptor activator of nuclear factor k-B ligand; RANKL: Nuclear factor of activated T cells c1; NFATc1: Tartrate-resistant acid phosphatase; TRAP: Cathepsin K; Ctsk: Dendritic-cell specific transmembrane protein; DC-STAMP: Osteoclast stimulatory transmembrane protein; OC-STAMP: a-minimal essential medium; a-MEM: cell counting kit-8; CCK-8: bone marrow cells; BMCs

\section{Declarations}

\section{Ethics approval and consent to participate}

Not applicable.

\section{Consent for publication}

Not applicable.

\section{Availability of data and materials}

Raw data, including imaging files, and reagents described in this study will be made available upon request to the corresponding author, but some reagents, which we bought, should be made directly to the companies.

\section{Competing interests}

The authors declare that they have no competing interests.

\section{Funding}

This work was supported by Grant-in-Aid for Scientific Research (A) no.15H01767 from the Japan Society for the Promotion of Science (JSPS).

\section{Authors' Contributions}

T.T., H.I., N.T., and M.U. designed the experiments. T.T. and H.I. performed the experiments. S.F. participated in cell culture. T.T., H.I., S.F., N.T., and M.U. analyzed the data. T.T., H.I., and M.U. wrote the manuscript. N.T. contributed to the development of the manuscript. All authors read and approved the final manuscript. 
Acknowledgments

We would like to thank Editage (www.editage.com) for English language editing.

\section{References}

1. Hadjidakis DJ, Androulakis II. Bone remodeling. Ann N Y Acad Sci. 2006; 1092:385-96.

2. Geusens P. The role of RANK ligand/osteoprotegerin in rheumatoid arthritis. Ther Adv Musculoskelet Dis. 2012; 4(4):225-33.

3. Hienz SA, Paliwal S, Ivanovski S. Mechanisms of Bone Resorption in Periodontitis. J Immunol Res. 2015; 2015:615486.

4. Lee ZH, Kim HH. Signal transduction by receptor activator of nuclear factor kappa B in osteoclasts. Biochem Biophys Res Commun. 2003; 30;305(2):211-4.

5. Boyle WJ, Simonet WS, Lacey DL. Osteoclast differentiation and activation. Nature. 2003; 15;423(6937):337-42.

6. Yagi M, Miyamoto T, Sawatani Y, Iwamoto $K$, Hosogane $N$ et al. DC-STAMP is essential for cell-cell fusion in osteoclasts and foreign body giant cells. J Exp Med. 2005; 202(3):345-51.

7. Miyamoto H, Suzuki T, Miyauchi Y, Iwasaki R, Kobayashi T et al. Osteoclast stimulatory transmembrane protein and dendritic cell-specific transmembrane protein cooperatively modulate cell-cell fusion to form osteoclasts and foreign body giant cells. J Bone Miner Res. 2012; 27(6):1289-97.

8. Abbaoui B, Riedl KM, Ralston RA, Thomas-Ahner JM, Schwartz SJ et al. Inhibition of bladder cancer by broccoli isothiocyanates sulforaphane and erucin: characterization, metabolism, and interconversion. Mol Nutr Food Res. 2012; 56(11):1675-87.

9. Prełowska M, Kaczyńska A, Herman-Antosiewicz A. 4-(Methylthio)butyl isothiocyanate inhibits the proliferation of breast cancer cells with different receptor status. Pharmacol Rep. 2017; 69(5):10591066.

10. Takagi T, Inoue H, Takahashi N, Katsumata-Tsuboi R, Uehara M. Sulforaphane inhibits osteoclast differentiation by suppressing the cell-cell fusion molecules DC-STAMP and OC-STAMP. Biochem Biophys Res Commun. 2017; 29;483(1):718-724.

11. Takagi T, Inoue H, Takahashi N, Katsumata-Tsuboi R, Uehara M. Sulforaphene attenuates multinucleation of pre-osteoclasts by suppressing expression of cell-cell fusion-associated genes DC-STAMP, OC-STAMP, and Atp6v0d2. Biosci Biotechnol Biochem. 2017; 81(6):1220-1223.

12. Kukita T, Wada N, Kukita A, Kakimoto T, Sandra F et al. RANKL-induced DC-STAMP is essential for osteoclastogenesis. J Exp Med. 2004; 4;200(7):941-6

13. Melchini A, Traka MH. Biological profile of erucin: a new promising anticancer agent from cruciferous vegetables. Toxins (Basel). 2010; 2(4):593-612. 
14. Callaway DA, Jiang JX. Reactive oxygen species and oxidative stress in osteoclastogenesis, skeletal aging and bone diseases. J Bone Miner Metab. 2015; 33(4):359-70.

15. Harris KE, Jeffery EH. Sulforaphane and erucin increase MRP1 and MRP2 in human carcinoma cell lines. J Nutr Biochem. 2008; 19(4):246-54.

16. Kim MJ, Kim SH, Lim SJ. Comparison of the Apoptosis-inducing Capability of Sulforaphane Analogues in Human Colon Cancer Cells. Anticancer Res. 2010; 30(9):3611-9.

\section{Figures}

(A)<smiles>CSCCCCN=C=S</smiles>

Erucin
(B)

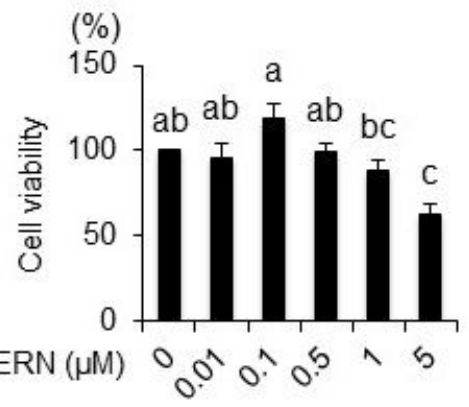

(C)

\begin{tabular}{|c|c|c|c|c|c|}
\hline 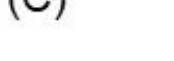 & & & $5(\mathrm{OH}$ & & \\
\hline ERN ( $\mu \mathrm{M})$ & 0 & 0.01 & 0.1 & 0.5 & 1 \\
\hline
\end{tabular}

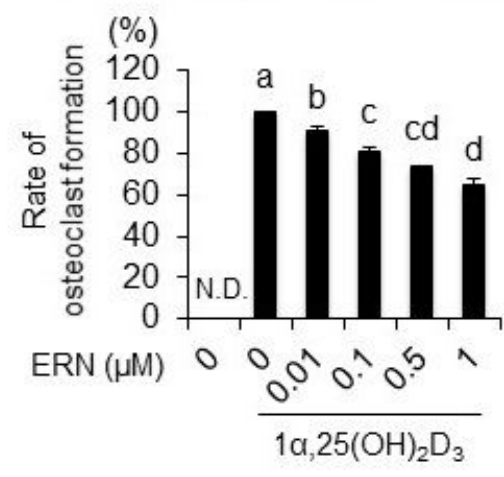

Fig. 1

\section{Figure 1}

Effects of ERN on osteoclast formation in BMCs Notes: (A) Chemical structure of ERN. (B) Effect of ERN on the cell viability of BMCs. The cytotoxic effect of ERN was evaluated using the CCK-8 assay. BMCs were treated with various concentrations of ERN (0-5 $\mu \mathrm{M})$ for 6 days. Cell viability is expressed as a percentage of the values obtained for untreated ERN-cells. (C) BMCs were cultured with various 
concentrations of ERN (0-1 $\mu \mathrm{M})$ in the presence of 1a,25(OH)2D3 (10-8 M) for 6 days. After incubation, the cells were fixed and stained for TRAP, a marker enzyme for osteoclast differentiation. TRAP-positive multinuclear cells ( $\geq 3$ nuclei) were counted. The effect of ERN on osteoclast differentiation is expressed as the rate of multinucleated osteoclast formation, with ERN-untreated cells set at $100 \%$. The data are expressed as the means \pm SE of three independent experiments $(n=3)$. Means marked with different letters are significantly different $(P<0.05)$.

(A)

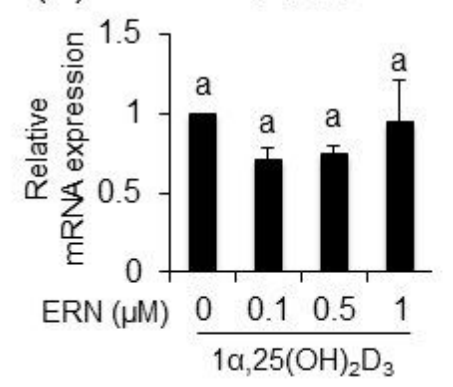

(D)

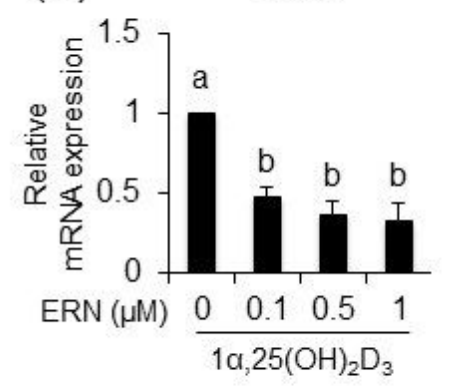

(B)

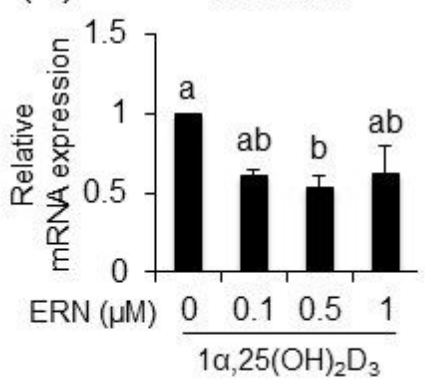

(E)

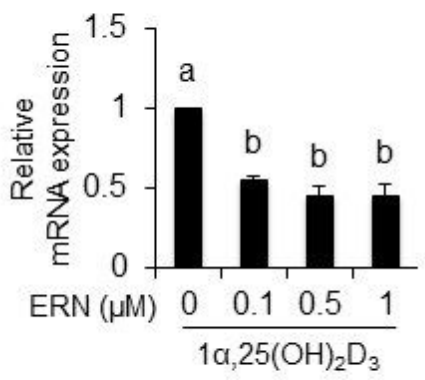

(C)

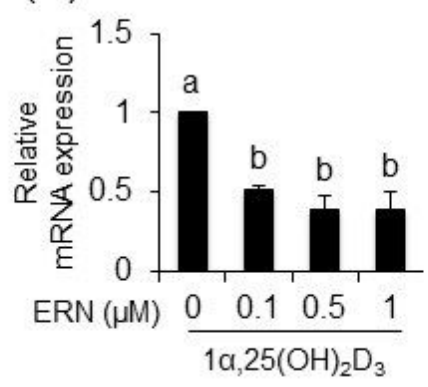

(F)

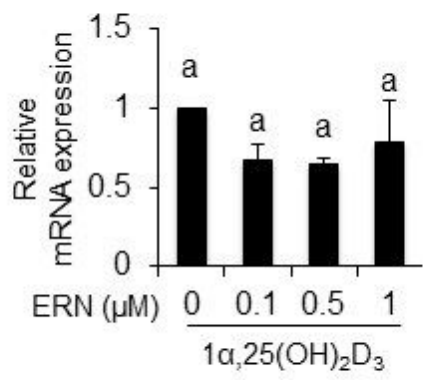

Fig. 2

\section{Figure 2}

Effects of ERN on expression of osteoclast-differentiation associated genes Notes: BMCs were cultured with various concentrations of ERN $(0-1 \mu \mathrm{M})$ in the presence of 1a,25(OH)2D3 (10-8 M) for 6 days. mRNA expression levels of c-Fos (A), NFATc1(B), TRAP(C), Ctsk (D), DC-STAMP(E), and OC-STAMP (F) were analyzed by real-time $P C R$, and the results were normalized to the expression of the $\beta$-actin-encoding ACTB gene. The data are expressed as the means \pm SE of three independent experiments $(n=3)$. Means marked with different letters are significantly different $(P<0.05)$. 
(A)

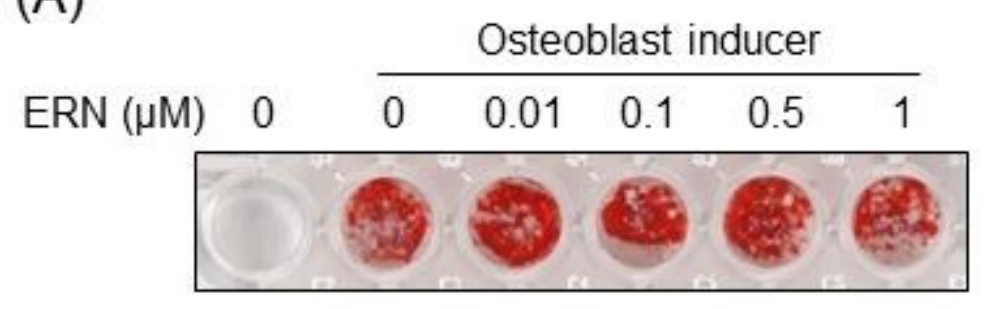

(B)

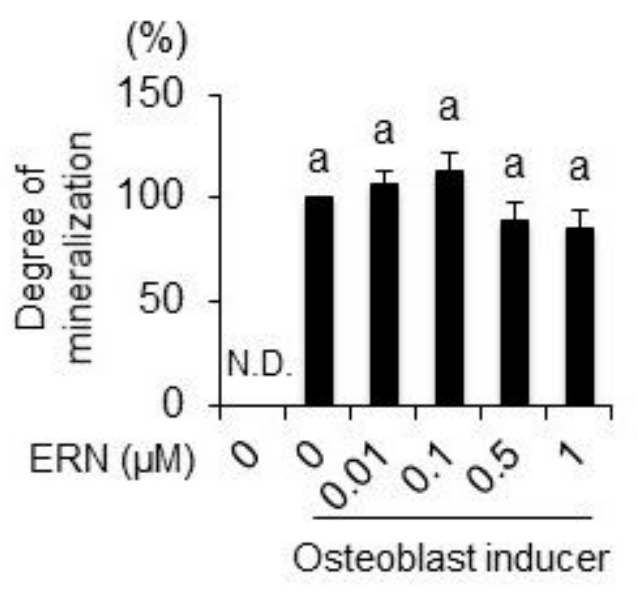

Fig. 3

Figure 3

Effects of ERN on osteoblast differentiation in BMCs Notes: (A) Mineralization of BMCs was assessed by alizarin red staining after 15 days of culture with osteoblast-inducer reagents (ascorbic acid, $\beta$ glycerophosphate, and hydrocortisone). (B) Staining activities were quantified by measure optical absorbance at $570 \mathrm{~nm}$ using a microplate reader. The effect of ERN on osteoblast differentiation is expressed as the degree of mineralization, with ERN-untreated cells set at $100 \%$. The data are expressed as the means \pm SE of multiple independent experiments $(n=6)$. Means marked with different letters are significantly different $(P<0.05)$.

\section{Supplementary Files}

This is a list of supplementary files associated with this preprint. Click to download.

- ARRIVEComplianceQuestionnaire20210811.pdf 
- SupplementalFigure1Fig.S1ERN.pptx

- SupplementalFigure1Fig.S1legendERNR.docx 